\title{
Measuring the cosmic bulk flow with 6dFGSv
}

\author{
Christina Magoulas $^{1,2}$, Christopher Springob ${ }^{2,3,4}$, Matthew Colless ${ }^{5}$, \\ Jeremy Mould ${ }^{4,6}$, John Lucey ${ }^{7}$, Pirin Erdoğdu ${ }^{8}$ and D. Heath Jones ${ }^{9}$ \\ ${ }^{1}$ Department of Astronomy, University of Cape Town, Private Bag X3, Rondebosch 7701, RSA, \\ email: cmagoulas@ast.uct.ac.za \\ ${ }^{2}$ Australian Astronomical Observatory, PO Box 915, North Ryde, NSW 1670, Australia, \\ ${ }^{3}$ ICRAR, The University of Western Australia, Crawley, WA 6009, Australia, \\ ${ }^{4}$ ARC Centre of Excellence for All-sky Astrophysics (CAASTRO), \\ ${ }^{5}$ RSAA, The Australian National University, Canberra, ACT 2611, Australia, \\ ${ }^{6}$ CAS, Swinburne University, Hawthorn, VIC 3122, Australia, \\ ${ }^{7}$ Department of Physics, University of Durham, Durham, DH1 3LE, UK, \\ ${ }^{8}$ Australian College of Kuwait, PO Box 1411, Safat 13015, Kuwait, \\ ${ }^{9}$ School of Physics, Monash University, Clayton, VIC 3800, Australia.
}

\begin{abstract}
While recent years have seen rapid growth in the number of galaxy peculiar velocity measurements, disagreements remain about the extent to which the peculiar velocity field - a tracer of the large-scale distribution of mass - agrees with both $\Lambda$ CDM expectations and with velocity field models derived from redshift surveys. The 6dF Galaxy Survey includes peculiar velocities for nearly 9000 early-type galaxies (6dFGSv), making it the largest and most homogeneous galaxy peculiar velocity sample to date. We have used the 6dFGS velocity field to determine the amplitude and scale of large-scale cosmic flows in the local universe and test standard cosmological models. We also compare the galaxy density and peculiar velocity fields to establish the distribution of dark and luminous matter and better constrain key cosmological parameters such as the redshift-space distortion parameter.
\end{abstract}

Keywords. galaxies: distances and redshifts, cosmology: observations - distance scale - largescale structure of universe

\section{Introduction}

Peculiar velocities are a direct, unbiased tracer of the underlying distribution of mass in the universe that are regulated by the scale and amplitude of fluctuations in the density field. The peculiar velocity field is therefore a powerful cosmological probe that can provide independent constraints on the parameters defining models of large-scale structure formation. It is sensitive to mass fluctuations on the largest scales, up to $\sim 100 h^{-1} \mathrm{Mpc}$, and remains the only such probe in the low-redshift universe.

The dipole moment of the peculiar velocity field, also known as the bulk flow, is a measure of the large-scale, coherent motion of matter. The most recent peculiar velocity studies consist of samples containing a large number of measurements (on the order of 5000) to reach a consensus in the scale of these flows and also establish whether they are consistent with the predictions of $\Lambda$ CDM. When averaged over a large enough volume, cosmological models predict that the bulk flow should approach the Hubble flow, commonly measured as a convergence to the rest frame of the cosmic microwave background (CMB). Whilst there is growing consensus in the direction of the bulk flow found by multiple studies, inconsistencies in the observed amplitude and scale still remain.

The distortion of the galaxy distribution in redshift-space by the peculiar velocity field can be characterized by the linear redshift distortion parameter, $\beta$. The form of this 
distortion is related to the growth rate of structure, $f=\Omega_{\mathrm{m}}^{0.55}$, by $\beta=f / b$, under the assumption that the galaxy density $\left(\delta_{\mathrm{g}}\right)$ and matter density $\left(\delta_{\mathrm{m}}\right)$ fluctuations are related by a linear bias parameter, $b$, such that $b=\delta_{\mathrm{g}} / \delta_{\mathrm{m}}$. By comparing the observed peculiar velocity field to a reconstructed prediction of the velocity field, we can determine the $\beta$ parameter, linking the total mass density and the bias in the distribution of galaxies relative to the underlying distribution of mass.

Using the Fundamental Plane (FP), we have measured distances and peculiar velocities for nearly 9000 6dFGS galaxies, as described in Springob et al. (2014), to form 6dFGSv - the largest and most homogeneous peculiar velocity sample to date. Using a maximumlikelihood (ML) approach, we measure the overall bulk galaxy motions from the 6dFGS velocity field for the local volume of the universe, finding broad agreement with the predicted density and velocity fields constructed from galaxy redshift surveys.

\section{The 6dFGSv peculiar velocity field}

In this study we use the 6dFGSv to analyse the peculiar velocity field of the southern hemisphere in the nearby $(z<0.055)$ universe. 6dFGSv provides the largest single sample of galaxy peculiar velocity measurements to date and is also more homogeneous than most previous large peculiar velocity samples. It is drawn from more than 11000 galaxies in the 6dFGS redshift sample (6dFGSz, Jones et al., 2009) for which we have FP data (see Campbell et al., 2014). The methods for deriving peculiar velocities with a Bayesian approach are detailed in Springob et al. (2014), where smoothed maps of the individual velocities are used to perform a cosmographical analysis.

In Magoulas et al. (in prep.), we develop a ML model for fitting the parameters defining the peculiar velocity field (such as $\beta$ and a bulk flow, $\mathbf{u}$ ), incorporating the velocity model reconstructions from Erdoğdu et al. (2006) and Branchini et al. (1999) as well as the 3D Gaussian FP model of Magoulas et al. (2012). We adopt a forward modelling approach: that is, we specify a velocity field model, apply it to the galaxies in the 6dFGSv sample, and determine whether this leads to a better ML fit of the FP in the observational parameter space, given the characterization of the FP as a 3D Gaussian distribution, and including the uncertainties in the observations and the correlations between them. We prefer the forward approach because in principle it allows simultaneous fitting of the FP and individual peculiar velocities, and in practice it provides better control over the errors and the biases they induce in the fit.

\section{Redshift-space distortion parameter}

We explore how well the peculiar velocities from 6dFGSv are traced by the reconstructions of both Erdoğdu et al. (2006) and Branchini et al. (1999). These models reconstruct the $3 \mathrm{D}$ density and velocity fields from redshift catalogues, and allow a velocity-velocity comparison with the observed velocity data to estimate the $\beta$ parameter.

Erdoğdu et al. (2006) reconstruct the density and velocity field from the 2MASS redshift survey out to a distance of $200 h^{-1} \mathrm{Mpc}$ (with an $8 h^{-1} \mathrm{Mpc}$ grid) generated assuming a fiducial value of $\beta_{\mathrm{fid}}=0.4$. The iterative model of Branchini et al. (1999) provides a complementary reconstruction of the local density and velocity field from the $\mathrm{PSCz}$ redshift survey out to a depth of $180 h^{-1} \mathrm{Mpc}$ with $\beta_{\text {fid }}=0.5$ (and a $\sim 2.8 h^{-1} \mathrm{Mpc}$ grid).

We compare the 6dFGSv sample with the reconstructed $2 \mathrm{MRS}$ velocity field (using the ML method described in Section 2) and obtain a fit of $\beta=0.30 \pm 0.08$ (see Table 1). This is in good agreement with other studies including Davis et al. (2011) who find $\beta=0.33 \pm 0.04$ and also Bilicki et al. (2011) who find $\beta=0.38 \pm 0.04$ (within $\sim 1.5 \sigma$ of our 
Table 1. Best-fit values (and rms scatter) of $\beta$ and $\mathbf{u}$ for the $6 \mathrm{dFGSv}$ sample $\left(N_{g}=8885\right.$ galaxies), fitting (1) $\beta$ only, (2) both $\beta$ and $\mathbf{u}_{\text {res }}$ and (3) $\mathbf{u}_{\text {tot }}$ only. For the total and residual bulk flow we also include the calculated values for the magnitude of the bulk flow $(|\mathbf{u}|)$ in $\mathrm{km}$ $\mathrm{s}^{-1}$ and the bulk flow direction $(l, b)$ in degrees.

\begin{tabular}{lcccccccc}
\hline Model & $v_{n}$ & $\begin{array}{c}\langle\beta\rangle \\
{[-]}\end{array}$ & $\begin{array}{c}u_{x} \\
{\left[\mathrm{~km} \mathrm{~s}^{-1}\right]}\end{array}$ & $\begin{array}{c}u_{y} \\
{\left[\mathrm{~km} \mathrm{~s}^{-1}\right]}\end{array}$ & $\begin{array}{c}u_{z} \\
{\left[\mathrm{~km} \mathrm{~s}^{-1}\right]}\end{array}$ & $\begin{array}{c}|\mathbf{u}| \\
{\left[\mathrm{km} \mathrm{s}^{-1}\right]}\end{array}$ & $\begin{array}{c}l \\
\circ\end{array}$ & $\begin{array}{c}b \\
\circ\end{array}$ \\
\hline$(1) \beta$ & $2 \mathrm{MRS}$ & $0.30 \pm 0.08$ & - & - & - & - & - & - \\
$(2) \beta, \mathbf{u}_{\text {res }}$ & $2 \mathrm{MRS}$ & $0.20 \pm 0.06$ & $-251 \pm 70$ & $+182 \pm 47$ & $-27 \pm 51$ & $324 \pm 45$ & $305 \pm 23$ & $35 \pm 13$ \\
\hline$(1) \beta$ & $\mathrm{PSCz}$ & $0.57 \pm 0.11$ & - & - & - & - & - & - \\
$(2) \beta, \mathbf{u}_{\text {res }}$ & $\mathrm{PSCz}$ & $0.33 \pm 0.10$ & $-274 \pm 75$ & $+106 \pm 55$ & $-9 \pm 49$ & $308 \pm 59$ & $312 \pm 23$ & $22 \pm 14$ \\
\hline$(3) \mathbf{u}_{\text {tot }}$ & - & - & $-358 \pm 80$ & $+118 \pm 53$ & $-81 \pm 56$ & $397 \pm 68$ & $303 \pm 8$ & $17 \pm 11$ \\
\hline
\end{tabular}

value) from a measurement of the clustering dipole. However Pike \& Hudson (2005) find a substantially larger $(\sim 2.8 \sigma)$ value for $\beta$ of $0.49 \pm 0.04$ from a comparison of their own 2MRS field reconstruction with observed velocities from three surveys. Such discrepancies may result from sensitivity to the assumed value of the linear bias in different samples the size and coverage of the samples, or the uncertainties in the peculiar velocities.

For the comparison with the PSCz reconstruction we find a best-fit $\beta$ of $0.57 \pm 0.11$ in agreement with previous PSCz comparisons by Nusser et al. (2001) with $\beta=0.5 \pm 0.1$ and also $\beta=0.55 \pm 0.06$ found by Radburn-Smith, Lucey \& Hudson (2004).

\section{The Local Bulk Flow Motion}

For the $6 \mathrm{dFGSv}$ volume, we measure a total bulk flow amplitude of $\left|u_{\text {tot }}\right|=397 \pm 68 \mathrm{~km}$ $\mathrm{s}^{-1}$ in the direction $(l, b)=\left(303^{\circ} \pm 8^{\circ}, 17^{\circ} \pm 11^{\circ}\right)$, larger but still comparable to previous measurements. In Figure 1 we compare the amplitude of the 6 dFGSv bulk flow to other measurements shown at the effective scale of the samples from which they were derived (approximately at the limiting radius of the sample or the error-weighted radius of a Gaussian window; although different volumes sample the local structures influencing the bulk flow in different ways). In general, the measured bulk motions of most studies are within the $90 \%$ range of theoretical expectations from $\Lambda$ CDM; although the $6 \mathrm{dFGSv}$ bulk flow amplitude, whilst outside the $90 \%$ confidence range, is still consistent with $\Lambda \mathrm{CDM}$ at the $1.5 \sigma$ level. The previous measurements are also consistent with a general trend of decreasing bulk flow amplitude at large radii.

The residual bulk flow amplitude from 6dFGSv, after accounting for the mass distribution in the 2MRS field, is $\left|u_{\text {res }}\right|=324 \pm 45 \mathrm{~km} \mathrm{~s}^{-1}$ in the direction $(l, b)=\left(305^{\circ} \pm 23^{\circ}\right.$, $\left.35^{\circ} \pm 13^{\circ}\right)$. In comparison, the residual bulk flow from the PSCz velocity field has a slightly smaller amplitude of $\left|u_{\text {res }}\right|=308 \pm 59 \mathrm{~km} \mathrm{~s}^{-1}$ in the direction $(l, b)=\left(312^{\circ} \pm 23^{\circ}\right.$, $22^{\circ} \pm 14^{\circ}$ ). Both of these residual flows (and the total bulk flow) tend to point in the direction of the Shapley supercluster at $\left(312^{\circ}, 31^{\circ}\right)$, suggesting Shapley is playing a dominant role in the motions of the $6 \mathrm{dFGSv}$ volume. The 6dFGSv residual bulk flow is also a significant proportion of the total bulk flow, suggesting the supercluster might be underestimated in the model volume, either because it is under-sampled at the edge of the survey or because it extends further out.

The results presented here are preliminary, and the methods used to fit the velocity field are still being improved. In future, we plan to extend the comparison of the $6 \mathrm{dFGSv}$ observations to other reconstructions including the predicted $2 \mathrm{M}++$ velocity field from Carrick et al. (submitted) and Hudson \& Carrick (this volume). 


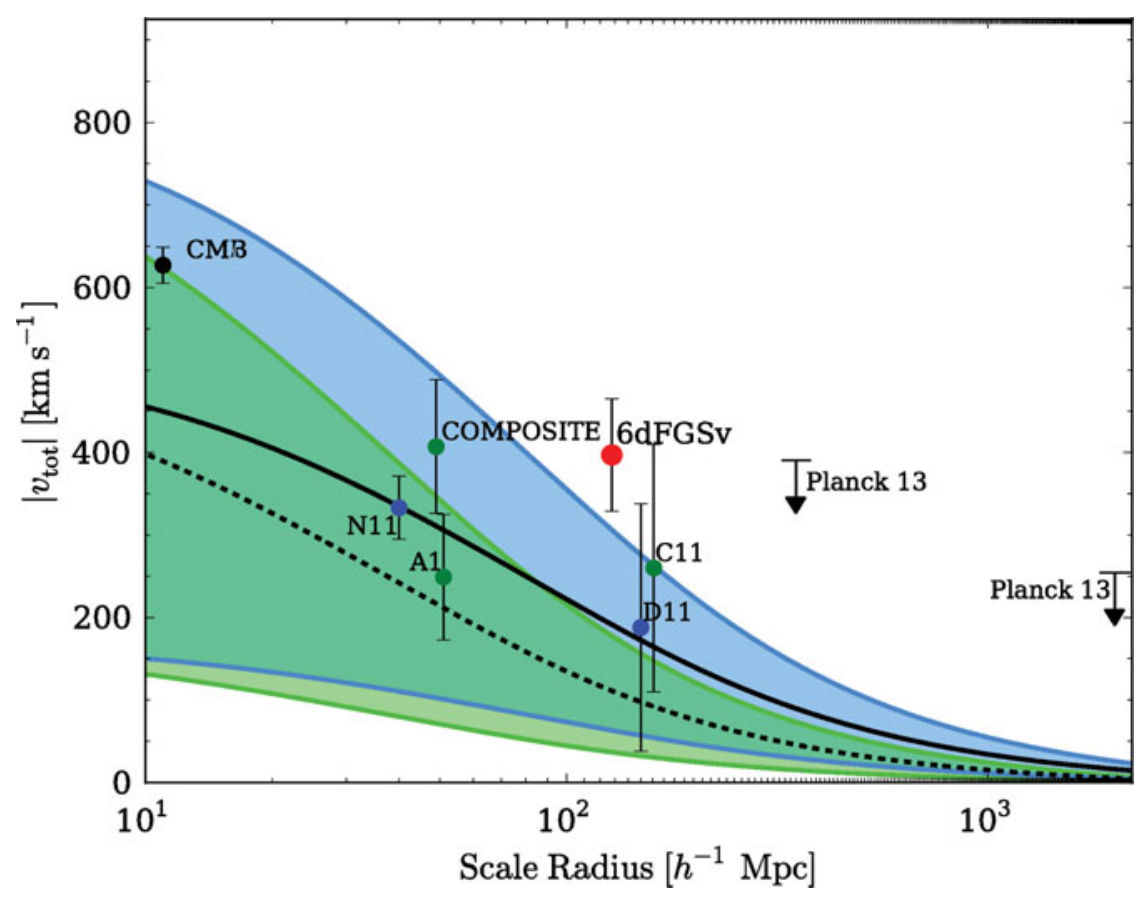

Figure 1. The bulk flow amplitude as a function of scale. The 6dFGSv bulk flow measurement is indicated in red at the radius of a sphere having the same volume as the hemispherical $6 \mathrm{dFGSv}$ survey. The predicted $\mathrm{rms}$ bulk flow in a flat $\Lambda \mathrm{CDM}$ model $\left(\Omega_{\mathrm{m}}=0.274, h=0.704\right.$ and $\sigma_{8}=0.811$ ) is shown as the solid (dashed) black line for a top-hat (Gaussian) window function. The light blue and green shadings around these lines are the $90 \%$ range of scatter from cosmic variance. Bulk flow measurements from recent studies, coloured according to the most appropriate window function (blue for top-hat, green for Gaussian), are shown for Nusser et al. (2011, N11), Watkins et al. (2009, COMPOSITE), Turnbull et al. (2012, A1), Colin et al. (2011, C11), Dai et al. (2011, D11) and Planck Collaboration (2013, Planck13) and also the Local Group motion with respect to the CMB (Kogut et al., 1993).

\section{References}

Bilicki, M., Chodorowski, M., Jarrett, T., \& Mamon, G. A. 2011, ApJ, 741, 31

Branchini, E. et al. 1999, MNRAS, 308, 1

Campbell, L. et al. 2014, MNRAS, 443, 1231

Colin, J., Mohayaee, R., Sarkar, S., \& Shafieloo, A. 2011, MNRAS, 414, 264

Dai, D.-C., Kinney, W. H., \& Stojkovic, D. 2011, JCAP, 4, 15

Davis, M. et al. 2011, MNRAS, 413, 2906

Erdoğdu, P. et al. 2006, MNRAS, 373, 45

Jones, D. H. et al. 2009, MNRAS, 399, 683

Kogut, A. et al. 1993, ApJ, 419, 1

Magoulas, C. et al. 2012, MNRAS, 427, 245

Nusser, A. \& Davis, M. 2011, ApJ, 736, 93

Nusser, A. et al. 2001, MNRAS, 320, 3

Pike, R. W. \& Hudson, M. J. 2005, ApJ, 635, 11

The Planck Collaboration 2013, ArXiv e-prints, 1303.5090

Radburn-Smith, D. J., Lucey, J. R., \& Hudson, M. J. 2004, MNRAS, 355, 4

Springob, C. M. et al. 2014, MNRAS, 445, 3

Turnbull, S. J. et al. 2012, MNRAS, 420, 447

Watkins, R., Feldman, H. A., \& Hudson, M. J. 2009, MNRAS, 392, 743 